\title{
A Bunch Stacking (Emittance Exchange) Scheme for a Muon Collider
}

\author{
Yasuo Fukui*, Alpher Garren, David Cline, Ping He, UCLA
}

\begin{abstract}
An emittance exchange scheme was studied with a simple simulation model where time-separated muon minibunches were stacked transversely through mini-bunch kicker, a solenoid delay channel, and a septum-type kicker. By stacking $\mathrm{N}$ mini muon bunches, we obtain the longitudinal phase space reduction of $1 / \mathrm{N}$ in maximum, which then be converted into the increased muon transverse phase space by a factor $\mathrm{N}$ in minimum due to the 6 dimensional phase space conservation. Limitations in the efficiency of the emittance exchange in this scheme was discussed. The emittance exchange is a key element to obtain the 10E-5 to $10 \mathrm{E}-6$ phase space cooling in the 6 dimensional muon phase space for a mu+mu- collider.
\end{abstract}

\section{INTRODUCTION}

The Higgs Factory will be the first $\mu^{+} \mu^{-}$collider to be built. Compared to a Neutrino Factory where muons decay in a straight section of a storage ring, the Higgs Factory requires more 6 dimensional phase space cooling of the muon beam by a factor of $10^{4-5}$. The Feasibility Studies I(Fermilab) and II(BNL) showed that those designs are feasible to build a neutrino Factory [3, 4].

In order to create the high luminosity in a collider, single high intensity $\mu^{+} / \mu^{-}$beam bunch will be transported into a collider where the many muon mini-bunch trains in the Neutrino Factory has to be combined into a single bunch.

The $\mu^{+} \mu^{-}$collider has been under designing for several year, and many reports have been made by the Neutrino Factory and the Muon Collider Collaboration. $[1,2]$ The on-going BNL targetry experiment E951 will reveal the feasibility of the proposed mercury jet target with the input proton beam power of multi MW. Several R\&D efforts on the components of the muon ionization cooling, $201 \mathrm{MHz}$ and $805 \mathrm{MHz}$ RF cavities, super conducting solenoid coils, and induction linac modules. Design of a cooling demonstration experiment is going on at Fermilab with the model of a cooling channel in a ring.

\section{GOALS}

Parameters of the general $\mu^{+} \mu^{-}$colliders including a Higgs Factory which was given in Reference [2].

6 dimensional normalized emittance is $1.7 \times 10^{-10}$ $\pi \mathrm{mm}$-mrad and the RMS beam length is $9.4 \mathrm{~cm}$, RMS $\Delta p / p$ is $0.01 \%$ in a Higgs Factory with the center of mass energy of $100 \mathrm{GeV}$. The proton beam power is $4 \mathrm{MW}$, and the luminosity is $2.2 \times 10^{31} \mathrm{~cm}^{-2} \mathrm{~s}^{-1}$.

\footnotetext{
*fukui@slac.stanford.edu
}

\section{STEPS FROM A NEUTRINO FACTORY TO A HIGGS FACTORY}

\subsection{Proton Bunches}

The requirement of the proton bunches is that a single proton bunch with the maximum available intensity hits target(s) within the $\gamma$ times the life time of the muons so that single $\mu^{+} / \mu^{-}$bunch can be collided in a collider ring. Reducing the number of proton bunches in the proton driver will reduce the effort to combine the muon long bunches later on. In case of the Neutrino Factory, the number of primary proton bunches was not restricted, because the muons can be injected into the decay storage ring any time, and the only issue was the total number of the decays of the muons in the straight section.

The high power proton driver design is given in References $[1,2]$. The primary proton power is estimated to be around $4 \mathrm{MW}$. Using the liquid metal target is the probable option to handle the severe energy deposition inside the target.

\subsection{Ring Cooler}

In order to add 6 dimensional phase space cooling to that in the neutrino Factory design, it is proposed to use a 6 dimensional ionization cooling ring which consists of cooling lattices, bending magnets, and wedge absorbers. The ionization cooling lattice is made of super conducting solenoid, RF cavities, and liquid Hydrogen absorber, The wedge absorbers are placed in high dispersion area so that $\Delta p / p$ in the longitudinal phase space is exchanged into the transverse phase space. The circumference of the ring is around $35 \mathrm{~m}$. Depending on the initial normalized emittance of the muons, the cooling ring is expected to generate the 6 dimensional phase space cooling factor of 30-100. [5]

Figure 1 shows a schematic diagram of the components of the Higgs Factory. The additional cooling ring is placed after the phase rotation channel and bunching section and before the linear cooling channel.

\subsection{Bunch Stacking Rings}

Stacking muon mini-bunches coming out of the 201 $\mathrm{MHz} \mathrm{RF}$ on top of each other naturally do the emittance exchange from the longitudinal phase space to the transverse phase space, besides the fact that getting a single muon bunch is the absolute must in the Higgs Factory.

Emittance exchange scheme by using transverse bunch stacking was first proposed by C. H. Kim [6]. The idea was then tested in a simulation by using a solenoid as a delay channel with pulse-by-pulse transverse bending which 


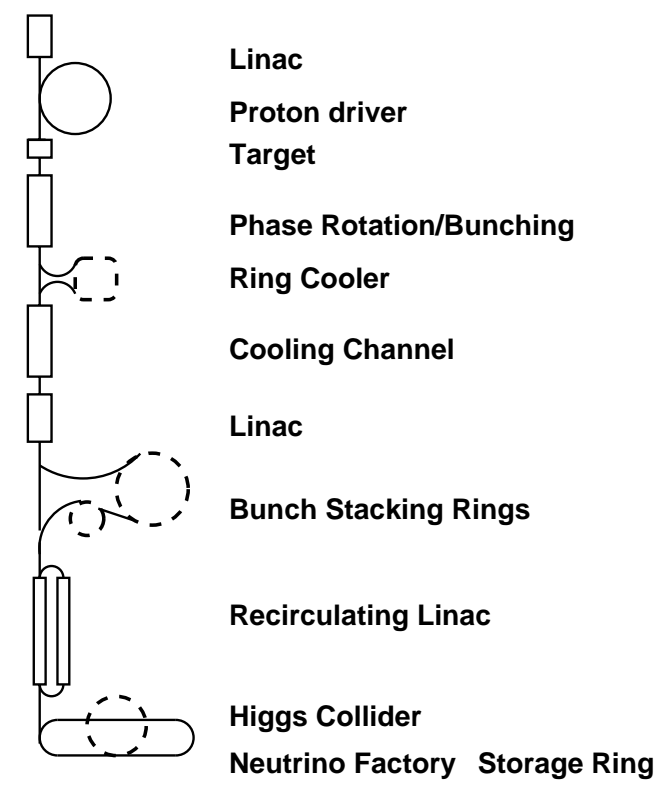

Figure 1: Schematic Diagram of the Higgs Factory and the Neutrino Factory

change the path length of beam bunches in the solenoid.

Figure 2 shows a schematic diagram of the transverse bunch stacking in a solenoid delay channel and in a bunch stacking ring.

In the Neutrino factory design, around 100 muon minibunches are created through the bunching channel and the ionization cooling channel with $201 \mathrm{MHz}$ RF cavities. We propose the transverse bunch stacking scheme by using an $1 \mathrm{GeV} 320 \mathrm{~m}$ ring with Lithium lens for the transverse phase space cooling, which contain all the 100 muon minibunches. by using a Fast Pulsed kicker magnet/EM filed, we then inject each mini bunches into an $1 \mathrm{GeV} 35 \mathrm{~m}$ smaller ring with Lithium lens element for the transverse cooling to stack the mini-bunches top on top each other so that we can get a single muon bunch. Figure 3 top plot shows the $\beta_{x}, \beta_{y}, \eta$, and the lattice component in the $320 \mathrm{~m}$ storage ring where a muon long bunch is contained with the $201 \mathrm{MHz}$ RF structure. Figure 3 bottom plot shows the $\beta_{x}$, $\beta_{y}, \eta$, and the lattice component in the $35 \mathrm{~m}$ storage ring where a muon single bunch is generated which then transfered in to the subsequent accelerator section. SYNCH simulation code was used to design the synchrotron rings with $320 \mathrm{~m}$ and $35 \mathrm{~m}$ circumferences and to analyze beam orbits in those rings. [9] Although the exact beam injection/extraction have not been computer-simulated, injecting muon mini-bunches from the $320 \mathrm{~m}$ ring into the $35 \mathrm{~m}$ ring is a challenging task. [8]

\section{CONCLUSION}

A scheme for a Higgs Factory has been designed by applying three major upgrades in the Neutrino Factory designs in the Feasibility studies I and II. The first is to use a
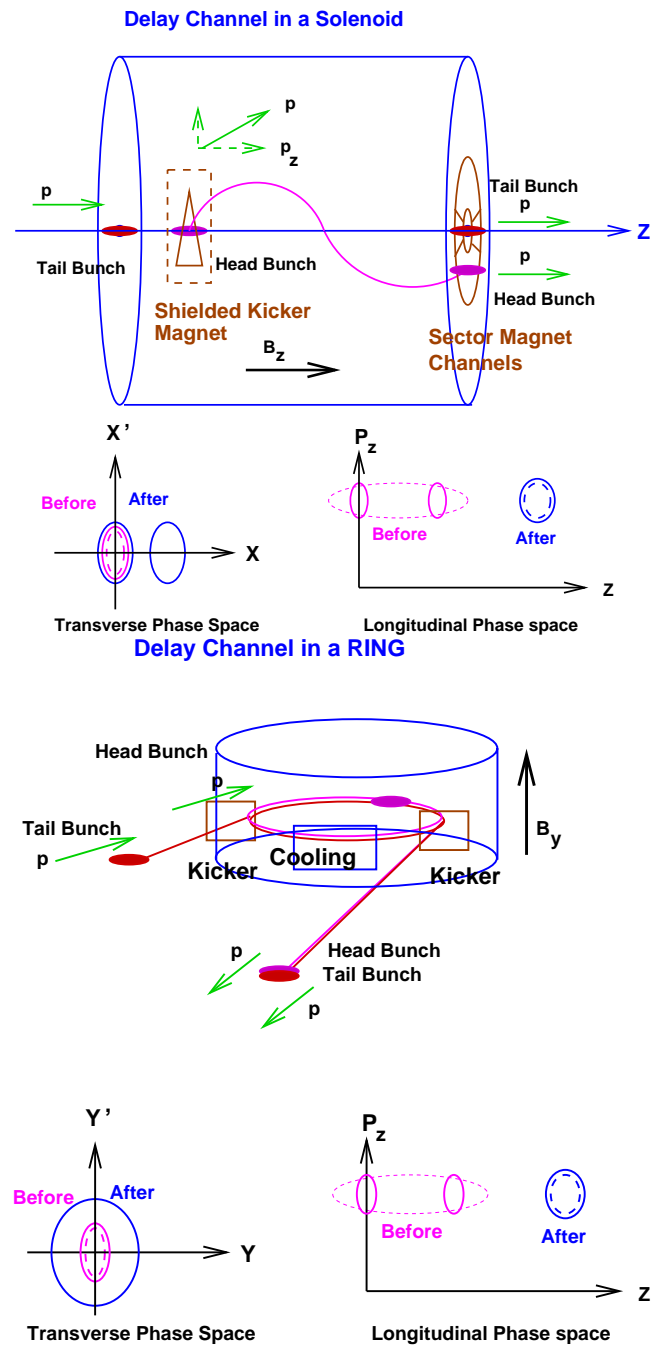

Figure 2: Schematic Diagram of the transverse bunch stacking in a solenoid delay channel and in a bunch stacking ring

single high intensity primary proton beams which are generated in a $4 \mathrm{MW}$ proton driver. The second is to add the 6 dimensional ionization cooling ring in between the phase rotation/bunching channel and the straight ionization cooling channel. The third is the transverse muon mini-bunch stacking by using a $320 \mathrm{~m}$ and $35 \mathrm{~m}$ synchrotron rings with Lithium lens for the transverse ionization cooling. A high intensity muon bunch will be generated there which then is accelerated and injected into a Higgs Collider storage ring. We need more detailed numerical simulation of the beam from the target to the collision point in the Higgs collider, where the goals are to obtain the high luminosity in the Higgs Factory.

\section{ACKNOWLEDGMENTS}

The authors wish to thank A Sessler, M. Zisman, J. Wurtele, W. Fawley, G. Penn, D. Neuffer, and V. Balbekov, for the useful discussions, R. B. Palmer, H. Kirk, R. Fernow 

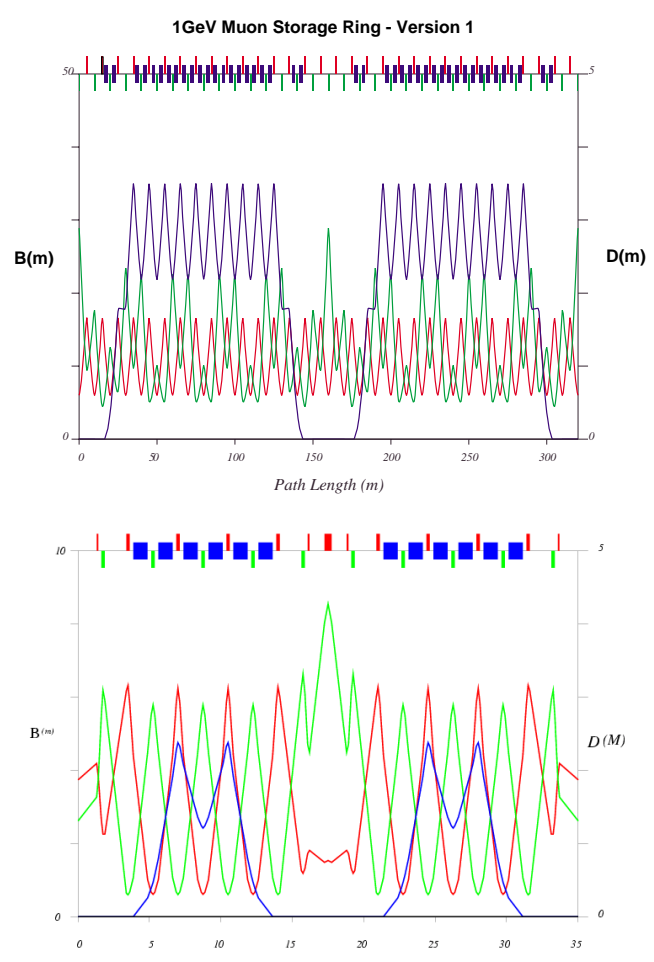

Figure 3: $\beta_{x}, \beta_{y}, \eta$, and the lattice component in $320 \mathrm{~m}$ ring(top) and in the $35 \mathrm{~m}$ ring(bottom)

and J. Gallardo, and, for their help and encouragement.

\section{REFERENCES}

[1] R. B. Palmer et al.,

" $\mu^{+} \mu^{-}$Collider: a Feasibility Study"

Snowmass96 workshop proceedings, BNL-52503,

Fermilab-Conf.-96.092, LBNL-38946

[2] C. M. Ankenbrandt et al.,

"Status of muon collider research and development and future plans"

Phys. Rev. ST Accel. Beams 2, 081001

[3] T. Anderson et al.,

"A Feasibility Study of a Neutrino Source Based on a Muon Storage Ring"

Fermilab report, March, 2000

[4] B. Palmer et al.,

"A BNL Feasibility Study II Report"

to be submitted, May, 2001

[5] V.Balbekov

"Ring Cooler Update"

Muon Collider Note 190, February, 2001

[6] C. H. Kim

"An Emittance Exchange Idea Using Transverse Bunch Stacking" Muon Collider Note 70, December, 1999

[7] R. Fernow

"A Simulation Code for Ionization Cooling of Muon Beams"

submitted to PAC99
[8] G. Schroder

"Fast Pulsed Magnet Systems"

CERN-SL-98-017(BT)

[9] A. Garren et al.,

"Synch(A Computer System for Synchrotron Design and Orbit Analysis) User's Guide"

SSSL-MAN-0030, 1993 